Bull. Korean Math. Soc. 50 (2013), No. 3, pp. 719-725

http://dx.doi.org/10.4134/BKMS.2013.50.3.719

\title{
A NOTE ON LPI DOMAINS
}

\author{
Kui Hu, Fanggui Wang, and Hanlin Chen
}

\begin{abstract}
A domain is called an LPI domain if every locally principal ideal is invertible. It is proved in this note that if $D$ is a LPI domain, then $D[X]$ is also an LPI domain. This fact gives a positive answer to an open question put forward by D. D. Anderson and M. Zafrullah.
\end{abstract}

\section{Introduction}

Throughout this note the ring $D$ will always be a domain with quotient field $K$. A flat $D$-module $M$ is called faithfully flat if $M \bigotimes_{D} N=0$ implies $N=0$ for any $D$-module $N$. It has been proved in [2] that a nonzero ideal $I$ of $D$ is faithfully flat if and only if $I$ is locally principal, that is, $I_{P}$ is a principle ideal of $D_{P}$ for any prime ideal $P$ of $D$. Let $A$ be a $D$-submodule of $K$. Define $A^{-1}=\{z \in K \mid z A \subseteq D\}$. A finitely generated ideal $J$ of $D$ is called a GV-ideal if $J^{-1}=D$. Denote by $G V(D)$ the set of GV-ideals of $D$. A domain $D$ is called an $\mathrm{H}$ domain if any ideal $I$ of $D$ with the property $I^{-1}=D$ contains a $\mathrm{GV}$-ideal. It is well-known that a finitely generated flat module over a domain is projective. Hence a finitely generated flat ideal over a domain is invertible. Domains with flat ideals invertible, called domains with property $\mathcal{P}$, were studied by Sally and Vasconcelos in [6] where they showed that if $D$ has property $\mathcal{P}$, then so does $D[X]$. In 1977, Glaz and Vasconcelos [2] asked whether a faithfully flat ideal $I$ of an $\mathrm{H}$ domain is projective, or equivalently, is finitely generated. This question has received a lot of attention. In 2009, D. D. Anderson and M. Zafrullah [1] introduced the notion of LPI domains. A domain $D$ is called an LPI domain if every nonzero locally principal ideal is invertible, or equivalently, if every faithfully flat ideal is finitely generated. Mori domains and therefore Noetherian domains are LPI domains. Obviously an LPI domain is a generalization of domains with the property $\mathcal{P}$, so is there an LPI domain that does not have property $\mathcal{P}$ ? The answer is simple: A nondiscrete rank one valuation domain is clearly an LPI domain but it does not satisfy the property $\mathcal{P}$, because the maximal ideal is flat but not invertible. Anderson and Zafrullah proved in [1] that if the polynomial ring $D[X]$ is an

Received February 3, 2012; Revised May 20, 2012.

2010 Mathematics Subject Classification. 13G05.

Key words and phrases. faithfully flat module, LPI domain, polynomial ring.

(ㄷ)2013 The Korean Mathematical Society 
LPI domain, then so is $D$ and that if $D$ is an integrally closed LPI domain, then $D[X]$ is an LPI domain. Then they asked consequently whether $D[X]$ is an LPI domain when $D$ is an LPI domain. In Section 1 of this note, we give a positive answer to this question.

Recently G. Picozza and F. Tartarone [5] put up with a counterexample to Glaz and Vasconcelos' question, that is, an H domain which is not an LPI domain. This result has inspired further investigation on LPI domains. In Section 2 of this note, we show that $D$ is an LPI domain if and only if every locally finitely generated and locally free submodule of $D^{n}$ of rank $n$ over $D$ is finitely generated. Thus, we give a module-theoretic description of LPI domains.

\section{Anderson-Zafrullah's question}

Let $f \in D[X]$. Denote by $c(f)$ the ideal of $D$ generated by coefficients of $f$, which is called the content of $f$. Let $J$ be an ideal of $D[X]$. Denote by $c(J)$ the set of coefficients of elements in $J$, which is an ideal of $D$ and is called the content of $J$.

Lemma 1.1. Let $J$ be a finitely generated ideal of $D[X]$. Then $J^{-1} \cap K[X]=$ $D[X]$ if and only if $c(J) \in \mathrm{GV}(D)$.

Proof. See [4, Lemma 4.2].

Lemma 1.2. Let $g \in D[X]$ with $g(0) \neq 0$. Then the ideal $J=\left(X^{n}, g\right) \in$ $\mathrm{GV}(D[X])$ for all $n \geqslant 1$.

Proof. We must prove that $J^{-1}=D[X]$. Let $\alpha \in J^{-1}$. Notice that $K[X]$ is a PID and the quotient field of $D[X]$ is also the quotient field of $K[X]$. Hence we may write $\alpha=\frac{h(X)}{f(X)}$, where $h(X)$ and $f(X)$ are in $K[X]$ and are relatively prime in $K[X]$. By the definition of $J^{-1}$, both $\frac{h(X) X^{n}}{f(X)}$ and $\frac{h(X) g}{f(X)}$ are elements of $D[X]$. Therefore $f(X) \mid X^{n}$ and $f(X) \mid g$ in the ring $K[X]$. Hence we get that $f(X)$ is a unit in $K[X]$, that is, $f(X) \in K$. Consequently $\alpha \in K[X]$, which implies that $J^{-1} \subset K[X]$. Clearly, $c(J)=D$ since $1 \in c(J)$. Hence $c(J)^{-1}=D$ (i.e., $c(J) \in \mathrm{GV}(D))$. Therefore, $J^{-1}=J^{-1} \cap K[X]=D[X]$ by Lemma 1.1, that is, $J \in \mathrm{GV}(R[X])$.

Lemma 1.3. Suppose $A$ is a flat ideal of $D$ and let $J$ be a GV-ideal of $D$. Suppose $z \in D$ with $J z \subseteq A$. Then $z \in A$.

Proof. See [6, Lemma 1.1].

Lemma 1.4. Let $A$ be a flat ideal of $D[X]$ such that $A$ is not contained in $X D[X]$. Then, for any $n \geqslant 1, A \bigcap X^{n} D[X]=X^{n} A$.

Proof. It is obvious that $A \bigcap X^{n} D[X] \supseteq X^{n} A$. We only need to prove the reverse inclusion. 
Let $h \in D[X]$ with $X^{n} h \in A$. Because $A$ is not contained in $X D[X]$, there exists an element $g \in A$, but $g \notin X D[X]$. Thus $g(0) \neq 0$. Thus we have $\left(X^{n}, g\right) h \subseteq A$. By Lemma 1.2 and Lemma 1.3 we get $h \in A$. Therefore, $A \cap X^{n} D[X] \subseteq X^{n} A$.

Lemma 1.5. Let $R$ be a commutative ring and let $I$ be a nilpotent ideal of $R$. Let $A$ be an $R$-module. Suppose $x_{1}, x_{2}, \ldots, x_{n} \in A$ such that $\left\{\overline{x_{1}}, \overline{x_{2}}, \ldots, \overline{x_{n}}\right\}$ is a generating set of $A / I A$. Then $\left\{x_{1}, x_{2}, \ldots, x_{n}\right\}$ is a generating set of $A$.

Proof. Let $B=R x_{1}+R x_{2}+\cdots+R x_{n}$. Then $A=I A+B$ by hypothesis. Thus we have $A / B=I(A / B)$. Since $I$ is nilpotent, we can assume that $I^{m}=0$ for some $m$. Therefore $A / B=I(A / B)=I^{2}(A / B)=\cdots=I^{m}(A / B)=0$, that imples $A=B$.

Theorem 1.6. If $A$ is a faithfully flat $D[X]$-module, then $A / X A$ must be a faithfully flat D-module.

Proof. Let $N$ be a $D$-module such that $A / X A \bigotimes_{D} N=0$. Then we have the isomorphism

$$
A / X A \bigotimes_{D} N \cong A \bigotimes_{D[X]} D[X] / X D[X] \bigotimes_{D} N=0 .
$$

Since $A$ is a faithfully flat $D[X]$-module, we get $N \cong D[X] / X D[X] \bigotimes_{D} N=0$. Therefore $A / X A$ is a faithfully flat $D$-module.

Lemma 1.7. Let $A$ be a flat ideal of $D[X]$. Then $A$ is finitely generated if and only if $c(A)$ is finitely generated.

Proof. See [6, Theorem 3.1].

In [6] Sally and Vasconcelos showed that if flat ideals of a domain $D$ are finitely generated, then flat ideals of $D[X]$ are also finitely generated. Now we begin to prove the main theorem. The trick of the following proof is due to Sally and Vasconcelos and what we do is a slight modification for our goal.

Theorem 1.8. If $D$ is an LPI domain, then $D[X]$ is also an LPI domain.

Proof. We only need to prove that faithfully flat ideals of the domain $D[X]$ are finitely generated. Let $A$ be a faithfully flat ideal of the domain $D[X]$. Since $\bigcap_{n=1}^{\infty} X^{n} D[X]=0$, we may assume $A \subseteq X^{k} D[X]$ for some $k \geqslant 0$, but $A \nsubseteq X^{k+1} D[X]$. Thus we have $A=X^{k} B$ for some ideal $B$ of $D[X]$ with $B \nsubseteq X D[X]$. Since $A \cong B$, by replacing $A$ with $B$ we can assume that $A \nsubseteq X D[X]$. Hence, for any $n \geqslant 1$, we have $A \cap X^{n} D[X]=X^{n} A$ by Lemma 1.4. Therefore, the natural homomorphism $A / X^{n} A \rightarrow D[X] / X^{n} D[X]$ is monomorphic.

By Lemma 1.4, the natural map $A / X A \rightarrow D[X] / X D[X]$ is a monomorphism. By Theorem 1.6, $A / X A$ is a faithfully flat ideal of $D \cong D[X] / X D[X]$. Hence $A / X A$ is finitely generated by hypothesis. Let $f_{1}, f_{2}, \ldots, f_{k} \in A$ such 
that $A=X A+\left(f_{1}, f_{2}, \ldots, f_{k}\right)$. Set $R_{n}=D[X] / X^{n} D[X]$. Denote by $\bar{f}$ the image of $f \in D[X]$ in $R_{n}$. Then in the ring $R_{n}=D[X] / X^{n} D[X]$, we have

$$
\bar{A}=\overline{X A}+\left(\overline{f_{1}}, \overline{f_{2}}, \ldots, \overline{f_{k}}\right) .
$$

Since $I=(\bar{X})$ is a nilpotent ideal of the ring $R_{n}=D[X] / X^{n} D[X]$, by Lemma 1.5 , we have

$$
A=X^{n} A+\left(f_{1}, f_{2}, \ldots, f_{k}\right) .
$$

Notice that this is true for all $n \geqslant 1$. Let $g$ be a nonzero element in $A$ and $\operatorname{deg}(g)=m$. For a sufficiently large integer $s$ such that $s>m$, we can find an element $h \in A$ and $h_{1}, h_{2}, \ldots, h_{k}$ of $D[X]$ such that

$$
g=X^{s} h+h_{1} f_{1}+\cdots+h_{k} f_{k} .
$$

Comparing coefficients of both side, we get that

$$
c(g) \subseteq c\left(f_{1}, f_{2}, \ldots, f_{k}\right) .
$$

Hence we have $c(A)=c\left(f_{1}, f_{2}, \ldots, f_{k}\right)$. Therefore, $c(A)$ is finitely generated. By Lemma 1.7, $A$ is finitely generated and this fact completes the proof of the theorem.

\section{Locally finitely generated and locally free modules}

We begin this section with a generalization of Glaz-Vasconcelos'result on faithfully flat ideal over a domain.

Proposition 2.1. Let $(D, \mathfrak{m})$ be a local domain and let $A$ be a $D$-submodule of $K$. Suppose $A$ is flat. Then either $A=\mathfrak{m} A$ or $A$ is a principal fractional ideal of $D$. In the later case, if $a \in A-\mathfrak{m} A$, then $A=(a)$.

Proof. If $A \neq \mathfrak{m} A$, take an element $a \in A$ but $a \notin \mathfrak{m} A$. For any $b \in A$, take a nonzero $s \in D$ such that $s a, s b \in D$. Thus $(s a) b-(s b) a=0$. Since $A$ is flat, there are elements $c_{i}, d_{i} \in D$ and $u_{i} \in A$ such that $a=\sum_{i} c_{i} u_{i}, b=\sum_{i} d_{i} u_{i}$, and $s a d_{i}=s b c_{i}$ for all $i$. Since $a \notin \mathfrak{m} A$, there is a $c_{i} \notin \mathfrak{m}$, say $c_{1}$. Thus $c_{1}$ is a unit. Hence $b=c_{1}^{-1} d_{1} a \in(a)$, which implies $A=(a)$.

Proposition 2.2. Let $A$ be a nonzero $D$-submodule of $K$. Then $A$ is faithfully flat if and only if $A_{\mathfrak{m}}$ is a principal fractional ideal of $D_{\mathfrak{m}}$ for any maximal ideal $\mathfrak{m}$ of $D$.

Proof. Suppose $A$ is faithfully flat and let $\mathfrak{m}$ be a maximal ideal of $D$. Hence $A / \mathfrak{m} A \neq 0$. Since $D / \mathfrak{m}$ is a field, we have $D_{\mathfrak{m}} / \mathfrak{m} D_{\mathfrak{m}} \cong D / \mathfrak{m}$. Consequently $A_{\mathfrak{m}} / \mathfrak{m} A_{\mathfrak{m}} \neq 0$, that is, $A_{\mathfrak{m}} \neq \mathfrak{m} A_{\mathfrak{m}}$. So $A_{\mathfrak{m}}$ is a principal fractional ideal of $D_{\mathfrak{m}}$ by Proposition 2.1 .

Conversely, if $A_{\mathfrak{m}}$ is a principal fractional ideal of $D_{\mathfrak{m}}$ for any maximal ideal $\mathfrak{m}$ of $D$, then $A$ is a flat $D$-module. Clearly, $A_{\mathfrak{m}} \neq \mathfrak{m} A_{\mathfrak{m}}$, and hence $A \neq \mathfrak{m} A$. This means that $A$ is faithfully flat. 
We know that a faithfully flat ideal of an LPI domain $D$ is finitely generated. The following example shows that a faithfully flat $D$-submodule of $K$ over an LPI domain $D$, even if locally principal, need not to be finitely generated.

Example 2.3. This example is to show that faithfully flat $D$-submodules of $K$ are not necessarily finitely generated. Let $A$ be a $\mathbb{Z}$-submodule in $\mathbb{Q}$ which is generated by the set $\left\{\frac{1}{p} \mid p\right.$ is a prime integer in $\left.\mathbb{Z}\right\}$. $\mathbb{Z}$ is Noetherian and therefore an LPI domain. It is easy to check that $A$ is faithfully flat and locally principal but $A$ is obviously not finitely generated.

Let $M$ be a locally finitely generated free module. Then $M$ is certainly torsion-free of finite $\operatorname{rank}$. If $\operatorname{rank}(M)=n$, then $M$ can be embedded in $K^{n}$. Naturally, we may view an element in $K^{n}$ as a row-vector over $K$. For $x_{i}=$ $\left(a_{i 1}, \ldots, a_{i n}\right) \in K^{n}, i=1, \ldots, n, A=\left(a_{i j}\right)$ is an $n \times n$ matrix over $K$. Define $\operatorname{det}\left(x_{1}, \ldots, x_{n}\right)=\operatorname{det}(A)$. Let $M$ be an $D$-submodule of $K^{n}$. Denote by $\operatorname{det}(M)$ the $D$-submodule of $K$ generated by the set $\left\{\operatorname{det}\left(x_{1}, \ldots, x_{n}\right) \mid x_{1}, \ldots, x_{n} \in M\right\}$ and call it the determinant of $M$. Clearly, if $M$ is finitely generated, then $\operatorname{det}(M)$ is a finitely generated fractional ideal of $D$; and if $M \subseteq D^{n}$, then $\operatorname{det}(M)$ is an ideal of $D$.

Proposition 2.4. Let $M$ be a D-submodule of $K^{n}$. Then we have:

(1) $\operatorname{det}(M)=0$ if and only if $\operatorname{rank}(M)<n$.

(2) If $N \subseteq M$, then $\operatorname{det}(N) \subseteq \operatorname{det}(M)$.

(3) Let $S$ be a multiplicative subset of D. Then $\operatorname{det}(M)_{S}=\operatorname{det}\left(M_{S}\right)$.

Proof. It is straightforward.

Theorem 2.5. Let $(D, \mathfrak{m})$ be a local domain and let $M$ be a D-submodule of $K^{n}$ of rank $n$. Then $M$ is finitely generated free if and only if $\operatorname{det}(M)$ is a nonzeo principal fractional ideal of $D$.

Proof. Suppose $M$ is finitely generated free. Let $\left\{x_{1}, \ldots, x_{n}\right\}$ be a basis of $M$ and write $a=\operatorname{det}\left(x_{1}, \ldots, x_{n}\right) \in K$. For any $y_{1}, \ldots, y_{n} \in M$, there is an $n \times n$ matrix $C$ over $D$ such that

$$
\left(\begin{array}{c}
y_{1} \\
\vdots \\
y_{n}
\end{array}\right)=C\left(\begin{array}{c}
x_{1} \\
\vdots \\
x_{n}
\end{array}\right) .
$$

Thus $\operatorname{det}\left(y_{1}, \ldots, y_{n}\right)=\operatorname{det}(C) a \in(a)$. It follows that $\operatorname{det}(M)=(a)$ is a principal fractional ideal of $D$.

Conversely, suppose $\operatorname{det}(M)=(a)$ is principal. Then we may write $a=$ $\sum_{i} r_{i} \operatorname{det}\left(x_{i 1}, \ldots, x_{i n}\right), r_{i} \in D, x_{i 1}, \ldots, x_{i n} \in M$. For each $i$, we have

$$
\operatorname{det}\left(x_{i 1}, \ldots, x_{i n}\right)=s_{i} a, s_{i} \in D \text {. }
$$

Since $D$ is a domain and $a \in K$, we have $\sum_{i} r_{i} s_{i}=1$. Because $D$ is local, we have that $s_{i}$ is a unit for some $i$. Therefore, we may assume $x_{1}, \ldots, x_{n} \in M$ with $\operatorname{det}\left(x_{1}, \ldots, x_{n}\right)=a$. Hence $x_{1}, \ldots, x_{n}$ are linearly independent over $D$. 
Let $x \in M$. Then $x=k_{1} x_{1}+\cdots+k_{n} x_{n}, k_{i} \in K, i=2, \ldots, n$. Since $\operatorname{det}\left(x, x_{2}, \ldots, x_{n}\right)=k_{1} a \in(a)$, we have $k_{1} \in D$. By the same argument, we have all $k_{i} \in D$. Therefore, $M$ is free with a basis $\left\{x_{1}, \ldots, x_{n}\right\}$.

Theorem 2.6. Let $M$ be a D-submodule of $K^{n}$ of rank $n$. Suppose $M$ is locally finitely generated and locally free. Then $\operatorname{det}(M)$ is faithfully flat.

Proof. Let $\mathfrak{m}$ be a maximal ideal of $D$. Then $M_{\mathfrak{m}}$ is free of rank $n$ by hypothesis. Hence $\operatorname{det}\left(M_{\mathfrak{m}}\right)=\operatorname{det}(M)_{\mathfrak{m}}$ is principal over $D_{\mathfrak{m}}$ by Proposition 2.4 and Theorem 2.5. Consequently $\operatorname{det}(M)$ is faithfully flat.

Theorem 2.7. Let $M$ be a D-submodule of $K^{n}$ of rank $n$. Suppose $M$ is locally finitely generated and locally free. If $\operatorname{det}(M)$ is finitely generated, then $M$ is finitely generated.

Proof. Let $\left\{\operatorname{det}\left(x_{i 1}, \ldots, x_{i n}\right) \mid i=1, \ldots, s\right\}$ be a generating set of $\operatorname{det}(M)$. For any maximal ideal $\mathfrak{m}$ of $D$, there is some $k(1 \leq k \leq s)$ such that $\operatorname{det}\left(x_{k 1}, \ldots, x_{k n}\right)$ is a basis of $\operatorname{det}\left(M_{\mathfrak{m}}\right)$ by Theorem 2.6 . Hence $\left\{x_{k 1}, \ldots, x_{k n}\right\}$ is a basis of $M_{\mathfrak{m}}$ by Theorem 2.5. Therefore, $\left\{x_{i j} \mid i=1, \ldots, s ; j=1, \ldots, n\right\}$ is a generating set of $M$.

Corollary 2.8. The following statements are equivalent for a domain $D$.

(1) $D$ is an LPI domain.

(2) Every locally finitely generated and locally free submodule of $D^{n}$ of rank $n$ over $D$ is finitely generated.

Proof. (1) $\Rightarrow(2)$ Let $M$ be a locally finitely generated and locally free submodule of $D^{n}$ of rank n over $D$. Thus $M$ can be embedded into $K^{n}$. By Theorem $2.6, \operatorname{det}(M)$ is a faithfully flat ideal of $D$. By hypothesis and Theorem 2.7, $M$ is finitely generated.

$(2) \Rightarrow(1)$ It is obvious since every nonzero locally principal ideal of $D$ is in fact a locally finitely generated and locally free submodule of $D$ of rank 1 over $D$.

\section{References}

[1] D. D. Anderson and M. Zafrullah, Integral domains in which nonzero locally principal ideals are invertible, Comm. Algebra 39 (2011), no. 3, 933-941.

[2] S. Glaz and W. V. Vasconcelos, Flat ideals. II, Manuscripta Math. 22 (1977), no. 4, $325-341$.

[3] , Flat ideals. III, Comm. Algebra 12 (1984), no. 1-2, 199-227.

[4] J. R. Hedstrom and E. G. Houston, Some remarks on star operations, J. Pure Appl. Algebra 18 (1980), no. 1, 37-44.

[5] G. Picozza and F. Tartarone, Flat ideals and stability in integral domains, J Algebra 324 (2010), no. 8, 1790-1802.

[6] J. D. Sally and W. V. Vasconcelos, Flat ideals I, Comm. Algebra 3 (1975), 531-543.

[7] M. Zafrullah, Flatness and invertibility of an ideal, Comm. Algebra 18 (1990), no. 7, $2151-2158$. 


\section{Kui Hu}

College of Sciences

Southwest University of Science and Technology

Mianyang Sichuan, 621010, P. R. China

E-mail address: hukui200418@163.com

FANGGUI WANG

College of Mathematics and Software Science

Sichuan Normal University

Chengdu, 610068, P. R. China

E-mail address: wangfg2004@163.com

Hanlin Chen

College of Sciences

Southwest University of Science and Technology

Mianyang Sichuan, 621010, P. R. China

E-mail address: chenhanlin@swust.edu.cn 